\begin{tabular}{|l|c|c|}
\hline \multicolumn{3}{|c|}{ DJS Vol. 38 (2017) 43-52 } \\
\hline 1969 & Delta Journal of Science & \\
\hline
\end{tabular}

\title{
A Method for Improving Rough Set Approximation Accuracy in terms of $\mathbf{j}$-Neighborhood Spaces
}

\author{
O. A. Embaby ${ }^{1}$ and Nadya. A. Toumi ${ }^{2}$ \\ 1 Department of Mathematics, Faculty of Science, Tanta University, EGYPT. \\ 2 Department of Mathematics, Faculty of Science, the $7^{\text {th }}$ of April University, Al-Zawia- LIBYA.
}

Email:_embaby@science.tanta.edu.eg

\begin{abstract}
This paper aims to introduce an effective method for improving rough set approximation accuracy. Considering the j-neighborhood space, the lower and upper approximation operators are defined and their fundamental properties are obtained. The approximations are constructed in four different approaches. Comparison between the accuracy of these four types of approximations is imposed and the best one is defined
\end{abstract}

Keywords: Rough Sets; j-Neighborhood Spaces; The Lower and Upper Rough Set Approximations.

\section{Introduction}

Rough set theory is a powerful tool for dealing with uncertainty, granularity, and incompleteness of knowledge in information systems. It is a mathematical approach which deals with vagueness by a pair of exact sets called the lower and upper approximation sets. These approximations correspond to minimal (resp. maximal) exact set contained in (resp. containing) the rough set. It was proved that the pair of lower and upper approximation operators induced by a reflexive and transitive binary relation is exactly a pair of interior and closure operators of a topology [6].

In Pawlak's original rough set theory [11], equivalence relation is a core concept which seems to be a very stringent condition that limits the application domain of the rough set theory. To solve this problem, several authors have generalized the notion of approximation operators by using arbitrary binary relations $[10,13,14,15,18,19,20$, and 22].

Lin [9] and Yao [21] studied rough sets using neighborhood systems for the interpretation of granules. M.E. Abd El-Monsef et al. [2] introduced mixed neighborhood systems to approximate rough sets. Lashin et al. [8] used a topology generated by right neighborhoods as a subbase and defined the lower and upper approximation operators by the interior and closure operators of this topology.

In 2014, Abd El-Monsef et al. [1] introduced the concept the $j$-neighborhood space which represents a generalized type of neighborhood spaces. Accordingly, we use this concept to define different types of the lower and upper approximation operators based on general binary relation. The lower and upper approximation operators are defined and their fundamental properties are obtained. The approximations are constructed in four different approaches. Comparison between the accuracy of these four types of approximations is imposed and the best one is defined.

\section{2. $j$-Neighborhood Spaces}

In this section, we give an exposition of the needed definitions. Also, we introduce a definition the lower and upper approximation operators in the $j$ neighborhood space and a definition of accuracy of the approximations of rough sets. 
Definition 1 Let $\mathcal{R}$ be an arbitrary binary relation on a non-empty finite set $U$.

The $j$-neighborhood of $x \in U\left(N_{j}(x)\right)$,

$$
j=r, \ell, i, u,\langle r\rangle,\langle\ell\rangle,\langle i\rangle,\langle u\rangle,
$$

can be defined as follows:

(i) $r$-neighborhood [4]:

(ii) $\ell$-neighborhood [5]:

$$
N_{r}(x)=\{y \in U \mid x R y\},
$$

(iii) $\langle r\rangle$-neighborhood [3]:

$$
N_{\langle r\rangle}(x)=\bigcap_{x \in N_{r}(y)} N_{r}(y),
$$

(iv) $\langle\ell\rangle$-neighborhood [3]:

$$
N_{\langle\ell\rangle}(x)=\bigcap_{x \in N_{\ell}(y)} N_{\ell}(y),
$$

(v) $i$-neighborhood [1]:

$$
N_{i}(x)=N_{r}(x) \cap N_{\ell}(x),
$$

(vi) $u$-neighborhood [1]:

$$
N_{u}(x)=N_{r}(x) \cup N_{\ell}(x),
$$

(vii) $\langle i\rangle$-neighborhood [1]:

$$
N_{\langle i\rangle}(x)=N_{\langle r\rangle}(x) \cap N_{\langle\ell\rangle}(x),
$$

(viii) $\langle u\rangle$-neighborhood [1]:

$$
N_{\langle u\rangle}(x)=N_{\langle r\rangle}(x) \cup N_{\langle\ell\rangle}(x) .
$$

Example 1 Let $\mathrm{U}=\{\mathrm{a}, \mathrm{b}, \mathrm{c}, \mathrm{d}, \mathrm{e}\}$ and $\mathcal{R}=$ $\{(a, b),(a, d),(b, b),(d, c),(d, e),(e, d),(e, e)\}$.

Thus we get

$$
\begin{aligned}
& N_{r}(a)=\{b, d\}, N_{\ell}(a)=\emptyset, N_{i}(a)=\emptyset, N_{u}(a)= \\
& \{b, d\} . N_{r}(b)=\{b\}, \quad N_{\ell}(b)=\{a, b\}, N_{i}(b)= \\
& \{b\}, N_{u}(b)=\{a, b\} . \\
& N_{r}(c)=\emptyset, \quad N_{\ell}(c)=\{d\}, N_{i}(c)=\emptyset, N_{u}(c)= \\
& \{d\} . N_{r}(d)=\{c, e\}, N_{\ell}(d)=\{a, e\}, N_{i}(d)= \\
& \{e\}, N_{u}(d)=\{a, c, e\} . \quad N_{r}(e)=\{d, e\}, N_{\ell}(e)= \\
& \{d, e\}, N_{i}(e)=\{d, e\}, N_{u}(e)=\{d, e\} .
\end{aligned}
$$

$N_{\langle r\rangle}(a)=\emptyset, N_{\langle\ell\rangle}(a)=\{a\}, N_{\langle i\rangle}(a)=\emptyset, N_{\langle u\rangle}(a)=$ $\{a\}$.

$N_{\langle r\rangle}(b)=\{b\}, N_{\langle\ell\rangle}(b)=\{a, b\}, N_{\langle i\rangle}(b)=$ $\{b\}, N_{\langle u\rangle}(b)=\{a, b\}$.

$N_{\langle r\rangle}(c)=\{c, e\}, N_{\langle\ell\rangle}(c)=\emptyset, N_{\langle i\rangle}(c)=\emptyset, N_{\langle u\rangle}(c)=$ $\{c, e\}$.

$N_{\langle r\rangle}(d)=\{d\}, N_{\langle\ell\rangle}(d)=\{d\}, N_{\langle i\rangle}(d)=$

$\{d\}, N_{\langle u\rangle}(d)=\{d\} . \quad N_{\langle r\rangle}(e)=\{e\}, N_{\langle\ell\rangle}(e)=$ $\{e\}, N_{\langle i\rangle}(e)=\{e\}, N_{\langle u\rangle}(e)=\{e\}$.

Definition 2 [1] Let $\mathcal{R}$ be an arbitrary binary relation on a non-empty finite set $U$ and the map $\xi_{j}: U \rightarrow$ $P(U)$ be a mapping which assigns for each $x$ in $U$ its $j$-neighborhood in $P(U)$, where $P(U)$ is the power set of $U$.The triple $\left(U, \mathcal{R}, \xi_{j}\right)$ is called a $j-$ neighborhood space.

Definition 3 Let $\left(U, R, \xi_{j}\right)$ be a $j$-neighborhood space and $A \subseteq U$. The $j$-lower and $j$-upper approximations of $A$ are defined respectively by

$$
\begin{aligned}
& \qquad \underline{R}_{j}(A)=\left\{p \in A: N_{j}(p) \neq \emptyset, N_{j}(p) \subseteq A\right\}, \\
& \bar{R}_{j}(A)=\mathrm{A} \cup\left\{\mathrm{p} \in \mathrm{A}^{\mathrm{c}}: N_{j}(p) \cap A \neq \phi\right\} \\
& \text { where } j=r, \ell, i, u,\langle r\rangle,\langle\ell\rangle,\langle i\rangle,\langle u\rangle .
\end{aligned}
$$

Definition 4 Let $\left(U, R, \xi_{j}\right)$ be a $j$-neighborhood space and $A \subseteq U$. The $j$-boundary, $j$-positive and $j$-negative regions of $A$ are defined respectively by

$$
\begin{gathered}
B_{j}(A)=\bar{R}_{j}(A)-\underline{R}_{j}(A), \\
P O S_{j}(A)=\underline{R}_{j}(A), \\
N E G_{j}(A)=U-\bar{R}_{j}(A),
\end{gathered}
$$

where $j=r, \ell, i, u,\langle r\rangle,\langle\ell\rangle,\langle i\rangle,\langle u\rangle$.

Definition 5 Let $\left(U, R, \xi_{j}\right)$ be

a $j$-neighborhood space. The $j$-accuracy of the approximations of $A \subseteq U$ is defined by

$$
\alpha_{j}(A)=\frac{\left|\underline{R}_{j}(A)\right|}{\left|\bar{R}_{j}(A)\right|}, \text { where }\left|\bar{R}_{j}(A)\right| \neq 0 \text {, }
$$

where $j=r, \ell, i, u,\langle r\rangle,\langle\ell\rangle,\langle i\rangle,\langle u\rangle$.

It is clear that, $0 \leq \alpha_{j}(A) \leq 1$ and if $\alpha_{j}(A)=1$ then $A$ is called $j$-definable (exact) set. Otherwise, it is called $j$-rough.

Example 2 Let $U=\{a, b, c, d\}$ and $\quad \mathcal{R}=$ $\{(a, c),(b, b),(c, a),(d, a)\}$. Thus we get

$\mathrm{N}_{r}(\mathrm{a})=\{\mathrm{c}\}, \mathrm{N}_{\ell}(\mathrm{a})=\{\mathrm{c}, \mathrm{d}\}, \mathrm{N}_{i}(\mathrm{a})=\{\mathrm{c}\}, \mathrm{N}_{u}(\mathrm{a})=$ $\{c, d\}$.

$\mathrm{N}_{r}(\mathrm{~b})=\{\mathrm{b}\}, \mathrm{N}_{\ell}(\mathrm{b})=\{\mathrm{b}\}, \mathrm{N}_{i}(\mathrm{~b})=\{\mathrm{b}\}, \mathrm{N}_{u}(\mathrm{~b})=\{\mathrm{b}\}$.

$\mathrm{N}_{r}(\mathrm{c})=\{\mathrm{a}\}, \mathrm{N}_{\ell}(\mathrm{c})=\{\mathrm{a}\}, \mathrm{N}_{i}(\mathrm{c})=\{\mathrm{a}\}, \mathrm{N}_{u}(\mathrm{c})=\{\mathrm{a}\}$.

$\mathrm{N}_{r}(\mathrm{~d})=\{\mathrm{a}\}, \mathrm{N}_{\ell}(\mathrm{d})=\phi, \mathrm{N}_{i}(\mathrm{~d})=\phi, \mathrm{N}_{u}(\mathrm{~d})=\{\mathrm{a}\}$.

$\mathrm{N}_{\langle r\rangle}(\mathrm{a})=\{\mathrm{a}\}, \mathrm{N}_{\langle\ell\rangle}(\mathrm{a})=\{\mathrm{a}\}, \mathrm{N}_{\langle i\rangle}(\mathrm{a})=\{\mathrm{a}\}, \mathrm{N}_{\langle u\rangle}(\mathrm{a})=$ $\{\mathrm{a}\}$.

$\mathrm{N}_{\langle r\rangle}(\mathrm{b})=\{\mathrm{b}\}, \mathrm{N}_{\langle\ell\rangle}(\mathrm{b})=\{\mathrm{b}\}, \mathrm{N}_{\langle i\rangle}(\mathrm{b})=\{\mathrm{b}\}, \mathrm{N}_{\langle u\rangle}(\mathrm{b})=$ $\{\mathrm{b}\}$. 
$\mathrm{N}_{\langle r\rangle}(\mathrm{c})=\{c\}, \mathrm{N}_{\langle\ell\rangle}(\mathrm{c})=\{\mathrm{c}, \mathrm{d}\}, \mathrm{N}_{\langle i\rangle}(\mathrm{c})=$

$\{c\}, N_{\langle u\rangle}(c)=\{c, d\}$.

$\mathrm{N}_{\langle r\rangle}(\mathrm{d})=\phi, \mathrm{N}_{\langle\ell\rangle}(\mathrm{d})=\{\mathrm{c}, \mathrm{d}\}, \mathrm{N}_{\langle i\rangle}(\mathrm{d})=\phi, \mathrm{N}_{\langle u\rangle}(\mathrm{d})=$

$\{c, d\}$.

Applying Definition 3, we have the following tables

Table $1: \underline{R}_{r}(A), \bar{R}_{r}(A), \underline{R}_{\ell}(A), \bar{R}_{\ell}(A), \underline{R}_{u}(A), \bar{R}_{u}(A), \underline{R}_{i}(A)$ and $\bar{R}_{i}(A)$ for all $A \subseteq U$.

\begin{tabular}{|c|c|c|c|c|c|c|c|c|}
\hline$A$ & $\underline{R}_{r}(A)$ & $\bar{R}_{r}(A)$ & $\underline{R}_{\ell}(A)$ & $\bar{R}_{\ell}(A)$ & $\underline{R}_{u}(A)$ & $\bar{R}_{u}(A)$ & $\underline{R}_{i}(A)$ & $\bar{R}_{i}(A)$ \\
\hline$\{a\}$ & $\varnothing$ & $\{a, c, d\}$ & $\varnothing$ & $\{a, c\}$ & $\varnothing$ & $\{a, c, d\}$ & $\varnothing$ & $\{a, c\}$ \\
\hline$\{b\}$ & $\{b\}$ & $\{b\}$ & $\{b\}$ & $\{b\}$ & $\{b\}$ & $\{b\}$ & $\{b\}$ & $\{b\}$ \\
\hline$\{c\}$ & $\varnothing$ & $\{a, c\}$ & $\varnothing$ & $\{a, c\}$ & $\varnothing$ & $\{a, c\}$ & $\varnothing$ & $\{a, c\}$ \\
\hline$\{d\}$ & $\varnothing$ & $\{d\}$ & $\varnothing$ & $\{a, c\}$ & $\varnothing$ & $\{a, d\}$ & $\varnothing$ & $\{d\}$ \\
\hline$\{a, b\}$ & $\{b\}$ & $U$ & $\{b\}$ & $\{a, b, c\}$ & $\{b\}$ & $U$ & $\{b\}$ & $\{a, b, c\}$ \\
\hline$\{a, c\}$ & $\{a, c\}$ & $\{a, c, d\}$ & $\{c\}$ & $\{a, c\}$ & $\{c\}$ & $\{a, c, d\}$ & $\{a, c\}$ & $\{a, c\}$ \\
\hline$\{a, d\}$ & $\{d\}$ & $\{a, c, d\}$ & $\emptyset$ & $\{a, c, d\}$ & $\{d\}$ & $\{a, c, d\}$ & $\varnothing$ & $\{a, c, d\}$ \\
\hline$\{b, c\}$ & $\{b\}$ & $\{a, b, c\}$ & $\{b\}$ & $\{a, b, c\}$ & $\{b\}$ & $\{a, b, c\}$ & $\{b\}$ & $\{a, b, c\}$ \\
\hline$\{b, d\}$ & $\{b\}$ & $\{b, d\}$ & $\{b\}$ & $\{a, b, d\}$ & $\{b\}$ & $\{a, b, d\}$ & $\{b\}$ & $\{b, d\}$ \\
\hline$\{c, d\}$ & $\varnothing$ & $\{a, c, d\}$ & $\emptyset$ & $\{a, c, d\}$ & $\emptyset$ & $\{a, c, d\}$ & $\varnothing$ & $\{a, c, d\}$ \\
\hline$\{a, b, c\}$ & $\{a, b, c\}$ & $U$ & $\{b, c\}$ & $\{a, b, c\}$ & $\{b, c\}$ & $U$ & $\{a, b, c\}$ & $\{a, b, c\}$ \\
\hline$\{a, b, d\}$ & $\{b, d\}$ & $U$ & $\{b\}$ & $U$ & $\{b, d\}$ & $U$ & $\{b\}$ & $U$ \\
\hline$\{a, c, d\}$ & $\{a, c, d\}$ & $\{a, c, d\}$ & $\{a, c\}$ & $\{a, c, d\}$ & $\{a, c, d\}$ & $\{a, c, d\}$ & $\{a, c\}$ & $\{a, c, d\}$ \\
\hline$\{b, c, d\}$ & $\{b\}$ & $U$ & $\{b\}$ & $U$ & $\{b\}$ & $U$ & $\{b\}$ & $U$ \\
\hline$U$ & $U$ & $U$ & $\{a, b, c\}$ & $U$ & $U$ & $U$ & $\{a, b, c\}$ & $U$ \\
\hline$\varnothing$ & $\emptyset$ & $\emptyset$ & $\emptyset$ & $\emptyset$ & $\emptyset$ & $\varnothing$ & $\varnothing$ & $\emptyset$ \\
\hline
\end{tabular}


Table 2: $\underline{R}_{\langle r\rangle}(A), \bar{R}_{\langle r\rangle}(A), \underline{R}_{\langle\ell\rangle}(A), \bar{R}_{\langle\ell\rangle}(A), \underline{R}_{\langle u\rangle}(A), \bar{R}_{\langle u\rangle}(A), \underline{R}_{\langle i\rangle}(A)$ and $\bar{R}_{\langle i\rangle}(A)$ for all $A \subseteq U$.

\begin{tabular}{|c|c|c|c|c|c|c|c|c|}
\hline$A$ & $\underline{R}_{\langle r\rangle}(A)$ & $\bar{R}_{\langle r\rangle}(A)$ & $\underline{R}_{\langle\ell\rangle}(A)$ & $\bar{R}_{\langle\ell\rangle}(A)$ & $\underline{R}_{\langle u\rangle}(A)$ & $\bar{R}_{\langle u\rangle}(A)$ & $\underline{R}_{\langle i\rangle}(A)$ & $\bar{R}_{\langle i\rangle}(A)$ \\
\hline$\{a\}$ & $\{a\}$ & $\{a\}$ & $\{a\}$ & $\{a\}$ & $\{a\}$ & $\{a\}$ & $\{a\}$ & $\{a\}$ \\
\hline$\{b\}$ & $\{b\}$ & $\{b\}$ & $\{b\}$ & $\{b\}$ & $\{b\}$ & $\{b\}$ & $\{b\}$ & $\{b\}$ \\
\hline$\{c\}$ & $\{c\}$ & $\{c\}$ & $\varnothing$ & $\{c, d\}$ & $\varnothing$ & $\{c, d\}$ & $\{c\}$ & $\{c\}$ \\
\hline$\{d\}$ & $\varnothing$ & $\{d\}$ & $\varnothing$ & $\{c, d\}$ & $\varnothing$ & $\{c, d\}$ & $\varnothing$ & $\{d\}$ \\
\hline$\{a, b\}$ & $\{a, b\}$ & $\{a, b\}$ & $\{a, b\}$ & $\{a, b\}$ & $\{a, b\}$ & $\{a, b\}$ & $\{a, b\}$ & $\{a, b\}$ \\
\hline$\{a, c\}$ & $\{a, c\}$ & $\{a, c\}$ & $\{a\}$ & $\{a, c, d\}$ & $\{a\}$ & $\{a, c, d\}$ & $\{a, c\}$ & $\{a, c\}$ \\
\hline$\{a, d\}$ & $\{a\}$ & $\{a, d\}$ & $\{a\}$ & $\{a, c, d\}$ & $\{a\}$ & $\{a, c, d\}$ & $\{a\}$ & $\{a, d\}$ \\
\hline$\{b, c\}$ & $\{b, c\}$ & $\{b, c\}$ & $\{b\}$ & $\{b, c, d\}$ & $\{b\}$ & $\{b, c, d\}$ & $\{b, c\}$ & $\{b, c\}$ \\
\hline$\{b, d\}$ & $\{b\}$ & $\{b, d\}$ & $\{b\}$ & $\{b, c, d\}$ & $\{b\}$ & $\{b, c, d\}$ & $\{b\}$ & $\{b, d\}$ \\
\hline$\{c, d\}$ & $\{c\}$ & $\{c, d\}$ & $\{c, d\}$ & $\{c, d\}$ & $\{c, d\}$ & $\{c, d\}$ & $\{c\}$ & $\{c, d\}$ \\
\hline$\{a, b, c\}$ & $\{a, b, c\}$ & $\{a, b, c\}$ & $\{a, b\}$ & $U$ & $\{a, b\}$ & $U$ & $\{a, b, c\}$ & $\{a, b, c\}$ \\
\hline$\{a, b, d\}$ & $\{a, b\}$ & $\{a, b, d\}$ & $\{a, b\}$ & $U$ & $\{a, b\}$ & $U$ & $\{a, b\}$ & $\{a, b, d\}$ \\
\hline$\{a, c, d\}$ & $\{a, c\}$ & $\{a, c, d\}$ & $\{a, c, d\}$ & $\{a, c, d\}$ & $\{a, c, d\}$ & $\{a, c, d\}$ & $\{a, c\}$ & $\{a, c, d\}$ \\
\hline$\{b, c, d\}$ & $\{b, c\}$ & $\{b, c, d\}$ & $\{b, c, d\}$ & $\{b, c, d\}$ & $\{b, c, d\}$ & $\{b, c, d\}$ & $\{b, c\}$ & $\{b, c, d\}$ \\
\hline$U$ & $\{a, b, c\}$ & $U$ & $U$ & $U$ & $U$ & $U$ & $\{a, b, c\}$ & $U$ \\
\hline$\varnothing$ & $\varnothing$ & $\varnothing$ & $\varnothing$ & $\varnothing$ & $\varnothing$ & $\varnothing$ & $\varnothing$ & $\varnothing$ \\
\hline
\end{tabular}


Table 3: $\alpha_{r}(A), \alpha_{\ell}(A), \alpha_{u}(A), \alpha_{i}(A), \alpha_{\langle r\rangle}(A), \alpha_{\langle\ell\rangle}(A), \alpha_{\langle u\rangle}(A)$ and $\alpha_{\langle i\rangle}(A)$ for all $A \subseteq U$.

\begin{tabular}{|c|c|c|c|c|c|c|c|c|}
\hline$A$ & $\alpha_{r}(A)$ & $\alpha_{\ell}(A)$ & $\alpha_{u}(A)$ & $\alpha_{i}(A)$ & $\alpha_{\langle r\rangle}(A)$ & $\alpha_{\langle\ell\rangle}(A)$ & $\alpha_{\langle u\rangle}(A)$ & $\alpha_{\langle i\rangle}(A)$ \\
\hline$\{a\}$ & 0 & 0 & 0 & 0 & 1 & 1 & 1 & 1 \\
\hline$\{b\}$ & 1 & 1 & 1 & 1 & 1 & 1 & 1 & 1 \\
\hline$\{c\}$ & 0 & 0 & 0 & 0 & 1 & 0 & 0 & 1 \\
\hline$\{d\}$ & 0 & 0 & 0 & 0 & 0 & 0 & 0 & 0 \\
\hline$\{a, b\}$ & $1 / 4$ & $1 / 3$ & $1 / 4$ & $1 / 3$ & 1 & 1 & 1 & 1 \\
\hline$\{a, c\}$ & $2 / 3$ & $1 / 2$ & $1 / 3$ & 1 & 1 & $1 / 3$ & $1 / 3$ & 1 \\
\hline$\{a, d\}$ & $1 / 3$ & 0 & $1 / 3$ & 0 & $1 / 2$ & $1 / 3$ & $1 / 3$ & $1 / 2$ \\
\hline$\{b, c\}$ & $1 / 3$ & $1 / 3$ & $1 / 3$ & $1 / 3$ & 1 & $1 / 3$ & $1 / 3$ & 1 \\
\hline$\{b, d\}$ & $1 / 2$ & $1 / 3$ & $1 / 3$ & $1 / 2$ & $1 / 2$ & $1 / 3$ & $1 / 3$ & $1 / 2$ \\
\hline$\{c, d\}$ & 0 & 0 & 0 & 0 & $1 / 2$ & 1 & 1 & $1 / 2$ \\
\hline$\{a, b, c\}$ & $3 / 4$ & $2 / 3$ & $1 / 2$ & 1 & 1 & $1 / 2$ & $1 / 2$ & 1 \\
\hline$\{a, b, d\}$ & $1 / 4$ & $1 / 4$ & $1 / 2$ & $1 / 4$ & $2 / 3$ & $1 / 2$ & $1 / 2$ & $2 / 3$ \\
\hline$\{a, c, d\}$ & 1 & $2 / 3$ & 1 & $2 / 3$ & $2 / 3$ & 1 & 1 & $2 / 3$ \\
\hline$\{b, c, d\}$ & $1 / 4$ & $1 / 4$ & $1 / 4$ & $1 / 4$ & $2 / 3$ & 1 & 1 & $2 / 3$ \\
\hline$U$ & 1 & $3 / 4$ & 1 & $3 / 4$ & $3 / 4$ & 1 & 1 & $3 / 4$ \\
\hline
\end{tabular}

neighborhood system of $p, \mathrm{~s}$ - neighborhood

3. The rough set approximations using three types of neighborhood systems

In this section, we introduce three types of neighborhood systems of any element $p$ in a $j$ neighborhood space $\left(\mathrm{U}, \mathcal{R}, \xi_{\mathrm{j}}\right)$, namely, the $\mathrm{k}$ - system of $p$ and t- neighborhood system of $p$. Making use of these three neighborhood systems, we define the lower and upper rough set approximations. Comparisons 
between the accuracy of these three types of approximations are obtained.

We proved that the best approximations are those based on the t- neighborhood systems. Moreover, the approximations based on the t- neighborhood systems are more accurate than the approximations which introduced in Definition 3.

Definition 6 Let $\left(\mathrm{U}, \mathcal{R}, \xi_{\mathrm{j}}\right)$ be a $j$-neighborhood space, and $p \in U$. Then

(i) the $\mathrm{k}$ - neighborhood system of $p$, sneighborhood system of $p$ and t- neighborhood system of $p$ are defined respectively by $N S_{k}(p)=\left\{N_{r}(p), N_{\ell}(p), N_{u}(p), N_{i}(p)\right\}$,

$N S_{s}(p)=\left\{N_{\langle r\rangle}(p), N_{\langle\ell\rangle}(p), N_{\langle u\rangle}(p), N_{\langle i\rangle}(p)\right\}$, $N S_{t}(p)=\left\{\begin{array}{c}N_{r}(p), N_{\ell}(p), N_{u}(p), N_{i}(p) \\ , N_{\langle r\rangle}(p), \\ N_{\langle\ell\rangle}(p), N_{\langle u\rangle}(p), N_{\langle i\rangle}(p)\end{array}\right\}$

(ii) every element of $N S_{k}(p), N S_{s}(p)$ and $N S_{t}(p)$ is called $N_{k}(p), N_{s}(p)$ and $N_{t}(p)$ respectively.

Definition 7 Let $\left(U, \mathcal{R}, \xi_{\mathrm{j}}\right)$ be a $j$-neighborhood space and $\mathrm{A} \subseteq \mathrm{U}$. Then

(i) The k-lower and k-upper approximations of $\mathrm{A}$ are defined respectively by

$$
\begin{gathered}
\underline{\mathcal{R}}_{k}(A)=\left\{p \in A: \exists N_{k}(p) \neq \phi, N_{k}(p) \subseteq A\right\}, \\
\overline{\mathcal{R}}_{k}(A)=A \cup\left\{p \in A^{c}: \forall N_{k}(p), N_{k}(p) \cap A \neq \phi\right\},
\end{gathered}
$$

(ii) The s-lower and s-upper approximations of $\mathrm{A}$ are defined respectively by

$$
\begin{gathered}
\underline{\mathcal{R}}_{\mathrm{s}}(\mathrm{A})=\left\{\mathrm{p} \in \mathrm{A}: \exists \mathrm{N}_{\mathrm{s}}(\mathrm{p}) \neq \phi, \mathrm{N}_{\mathrm{s}}(\mathrm{p}) \subseteq \mathrm{A}\right\}, \\
\overline{\mathcal{R}}_{S}(A)=A \cup\left\{p \in A^{c}: \forall N_{s}(p), N_{s}(p) \cap A \neq \phi\right\},
\end{gathered}
$$

(iii) The t-lower and t-upper approximations of $\mathrm{A}$ are defined respectively by

$$
\begin{gathered}
\underline{\mathcal{R}}_{t}(A)=\left\{p \in A: \exists N_{t}(p) \neq \phi, N_{t}(p) \subseteq A\right\} \\
\overline{\mathcal{R}}_{t}(A)=A \cup\left\{p \in A^{c}: \forall N_{t}(p), N_{t}(p) \cap A \neq \phi\right\} .
\end{gathered}
$$

Definition 8 Let $\left(\mathrm{U}, \mathcal{R}, \xi_{\mathrm{j}}\right)$ be a $j$-neighborhood space, and $\mathrm{A} \subseteq \mathrm{U}$. Then:

(i) The boundary, positive and negative regions of $\mathrm{A}$ using k-neighborhood system are defined respectively by

$$
\begin{gathered}
B_{k}(A)=\overline{\mathcal{R}}_{\mathrm{k}}(\mathrm{A})-\underline{\mathcal{R}}_{\mathrm{k}}(\mathrm{A}), \\
\operatorname{POS}_{k}(A)=\underline{\mathcal{R}}_{\mathrm{k}}(\mathrm{A}) \\
N E G_{k}(A)=\mathrm{U}-\overline{\mathcal{R}}_{\mathrm{k}}(\mathrm{A})
\end{gathered}
$$

(ii) The boundary, positive and negative regions of $\mathrm{A}$ using s-neighborhood system are defined respectively by

$$
\begin{gathered}
B_{S}(A)=\overline{\mathcal{R}}_{\mathrm{S}}(\mathrm{A})-\underline{\mathcal{R}}_{\mathrm{S}}(\mathrm{A}), \\
P O S_{S}(A)=\underline{\mathcal{R}}_{\mathrm{S}}(\mathrm{A}), \\
N E G_{S}(A)=\mathrm{U}-\overline{\mathcal{R}}_{\mathrm{S}}(\mathrm{A}),
\end{gathered}
$$

(iii) The boundary, positive and negative regions of $\mathrm{A}$ using t-neighborhood system are defined respectively by

$$
\begin{gathered}
B_{\mathrm{t}}(\mathrm{A})=\overline{\mathcal{R}}_{\mathrm{t}}(\mathrm{A})-\underline{\mathcal{R}}_{\mathrm{t}}(\mathrm{A}), \\
\operatorname{POS}_{t}(A)=\underline{\mathcal{R}}_{\mathrm{t}}(\mathrm{A}), \\
N E G_{t}(A)=\mathrm{U}-\overline{\mathcal{R}}_{\mathrm{t}}(\mathrm{A}) .
\end{gathered}
$$

Definition 9 Let $\left(U, R, \xi_{j}\right)$ be a $j$-neighborhood space and $\mathrm{A} \subseteq \mathrm{U}$. Then the accuracy of the approximations of a subset $A$ using k-neighborhood system, sneighborhood system and t-neighborhood system are defined respectively by

$$
\begin{aligned}
& \alpha_{k}(A)=\frac{\left|\underline{\mathcal{R}}_{k}(A)\right|}{\left|\overline{\mathcal{R}}_{k}(A)\right|}, \\
& \alpha_{s}(A)=\frac{\left|\underline{\mathcal{R}}_{s}(A)\right|}{\left|\overline{\mathcal{R}}_{s}(A)\right|}, \\
& \alpha_{t}(A)=\frac{\left|\underline{\mathcal{R}}_{t}(A)\right|}{\left|\overline{\mathcal{R}}_{t}(A)\right|} .
\end{aligned}
$$

Where $\left|\overline{\mathcal{R}}_{\mathrm{k}}(\mathrm{A})\right|,\left|\overline{\mathcal{R}}_{\mathrm{s}}(\mathrm{A})\right|$ and $\left|\overline{\mathcal{R}}_{\mathrm{t}}(\mathrm{A})\right| \neq 0$.

It is Obvious that, $0 \leq \alpha_{\mathrm{k}}(\mathrm{A}) \leq 1,0 \leq \alpha_{\mathrm{s}}(\mathrm{A}) \leq 1$ and $0 \leq \alpha_{t}(\mathrm{~A}) \leq 1$. 


\begin{tabular}{|c|c|c|c|c|c|c|}
\hline \multicolumn{7}{|c|}{ Table 4: $\underline{R}_{k}(A), \bar{R}_{k}(A), \underline{R}_{s}(A), \bar{R}_{s}(A), \underline{R}_{t}(A), \bar{R}_{t}(A)$ for all $A \subseteq U$} \\
\hline$A$ & $\underline{R}_{k}(A)$ & $\bar{R}_{k}(A)$ & $\underline{R}_{S}(A)$ & $\bar{R}_{S}(A)$ & $\underline{R}_{t}(A)$ & $\bar{R}_{t}(A)$ \\
\hline$\{a\}$ & $\varnothing$ & $\{a, c\}$ & $\{a\}$ & $\{a\}$ & $\{a\}$ & $\{a\}$ \\
\hline$\{b\}$ & $\{b\}$ & $\{b\}$ & $\{b\}$ & $\{b\}$ & $\{b\}$ & $\{b\}$ \\
\hline$\{c\}$ & $\varnothing$ & $\{a, c\}$ & $\{c\}$ & $\{c\}$ & $\{c\}$ & $\{c\}$ \\
\hline$\{d\}$ & $\varnothing$ & $\{d\}$ & $\varnothing$ & $\{d\}$ & $\varnothing$ & $\{d\}$ \\
\hline$\{a, b\}$ & $\{b\}$ & $\{a, b, c\}$ & $\{a, b\}$ & $\{a, b\}$ & $\{a, b\}$ & $\{a, b\}$ \\
\hline$\{a, c\}$ & $\{a, c\}$ & $\{a, c\}$ & $\{a, c\}$ & $\{a, c\}$ & $\{a, c\}$ & $\{a, c\}$ \\
\hline$\{a, d\}$ & $\{d\}$ & $\{a, c, d\}$ & $\{a\}$ & $\{a, d\}$ & $\{a, d\}$ & $\{a, d\}$ \\
\hline$\{b, c\}$ & $\{b\}$ & $\{a, b, c\}$ & $\{b, c\}$ & $\{b, c\}$ & $\{b, c\}$ & $\{b, c\}$ \\
\hline$\{b, d\}$ & $\{b\}$ & $\{b, d\}$ & $\{b\}$ & $\{b, d\}$ & $\{b\}$ & $\{b, d\}$ \\
\hline$\{c, d\}$ & $\varnothing$ & $\{a, c, d\}$ & $\{c, d\}$ & $\{c, d\}$ & $\{c, d\}$ & $\{c, d\}$ \\
\hline$\{a, b, c\}$ & $\{a, b, c\}$ & $\{a, b, c\}$ & $\{a, b, c\}$ & $\{a, b, c\}$ & $\{a, b, c\}$ & $\{a, b, c\}$ \\
\hline$\{a, b, d\}$ & $\{b, d\}$ & $U$ & $\{a, b\}$ & $\{a, b, d\}$ & $\{a, b, d\}$ & $\{a, b, d\}$ \\
\hline$\{a, c, d\}$ & $\{a, c, d\}$ & $\{a, c, d\}$ & $\{a, c, d\}$ & $\{a, c, d\}$ & $\{a, c, d\}$ & $\{a, c, d\}$ \\
\hline$\{b, c, d\}$ & $\{b\}$ & $U$ & $\{b, c, d\}$ & $\{b, c, d\}$ & $\{b, c, d\}$ & $\{b, c, d\}$ \\
\hline$U$ & $U$ & $U$ & $U$ & $U$ & $U$ & $U$ \\
\hline$\varnothing$ & $\varnothing$ & $\varnothing$ & $\varnothing$ & $\varnothing$ & $\varnothing$ & $\varnothing$ \\
\hline
\end{tabular}


Moreover, if $\alpha_{k}(A)=1 \quad$ (respectively $\alpha_{s}(A)=1$ or $\left.\alpha_{t}(A)=1\right)$, then $A$ is called k-definable(respectively s-definable or t-definable) set. Otherwise, it is called krough (respectively s-rough or t- rough) set.

Example 3 According to Example 2, we have the neighborhood systems

$$
\begin{gathered}
\begin{aligned}
N S_{k}(a)=\{\{c\},\{c, d\}\}, N S_{k}(b)=\{\{\mathrm{b}\}\}, \mathrm{NS}_{\mathrm{k}}(\mathrm{c}) \\
=\{\{a\}\}, N S_{k}(d)=\{\emptyset,\{\mathrm{a}\}\}
\end{aligned} \\
\begin{aligned}
N S_{s}(a)=\{\{a\}\}, \mathrm{NS}_{\mathrm{s}}(b)=\{\{b\}\}, N S_{s}(c) \\
=\{\{\mathrm{c}\},\{\mathrm{c}, \mathrm{d}\}\}, \mathrm{NS}_{\mathrm{s}}\{\mathrm{d}\}=\{\varnothing,\{\mathrm{c}, \mathrm{d}\}\}
\end{aligned} \\
\begin{aligned}
N S_{t}(a)=\{\{a\},\{c\},\{c, d\}\}, N S_{t}(b)=\{\{b\}\}, \mathrm{NS}_{\mathrm{t}}(c) \\
=\{\{a\},\{c\},\{c, d\}\},
\end{aligned} \\
\begin{aligned}
N S_{t}(d)=\{\varnothing,\{a\},\{c, d\}\} .
\end{aligned}
\end{gathered}
$$

Proposition 1. Let $\left(U, \mathcal{R}, \xi_{j}\right)$ be a $j$-neighborhood space and $\mathrm{A} \subseteq \mathrm{U}$. Then

(i) $\underline{\mathcal{R}}_{\mathrm{j}}(\mathrm{A}) \subseteq \underline{\mathcal{R}}_{\mathrm{t}}(\mathrm{A})$,

(ii) $\overline{\mathcal{R}}_{\mathrm{t}}(\mathrm{A}) \subseteq \overline{\mathcal{R}}_{\mathrm{j}}(\mathrm{A})$,

(iii) $\mathcal{B}_{\mathrm{t}}(\mathrm{A}) \subseteq \mathcal{B}_{\mathrm{j}}(\mathrm{A})$,

(iv) $\alpha_{\mathrm{j}}(\mathrm{A}) \leq \alpha_{\mathrm{t}}(\mathrm{A})$.

Proof. (i) Let $p \in \underline{\mathcal{R}}_{\mathrm{j}}(\mathrm{A})$, then $\mathrm{p} \in \mathrm{A}$ such that $N_{j}(p) \neq \varnothing, N_{j}(p) \subseteq A$. Thus $\mathrm{p} \in \mathrm{A}$ such that $\exists N_{t}(p) \neq \emptyset, N_{t}(p) \subseteq A$. Hence $p \in \underline{\mathcal{R}}_{\mathrm{t}}(\mathrm{A})$ and so $\underline{\mathcal{R}}_{\mathrm{j}}(\mathrm{A}) \subseteq \underline{\mathcal{R}}_{\mathrm{t}}(\mathrm{A})$.

(ii) Let $p \notin \overline{\mathcal{R}}_{\mathrm{j}}(\mathrm{A})$, then $\mathrm{p} \in \mathrm{A}^{\mathrm{C}}$ and $N_{j}(p) \cap A=\emptyset$. Thus $\mathrm{p} \in \mathrm{A}^{\mathrm{C}}$ and $\exists N_{t}(p)$,

$N_{t}(p) \cap A=\emptyset$. So, $p \notin \overline{\mathcal{R}}_{\mathrm{t}}(\mathrm{A})$. Therefore, $\overline{\mathcal{R}}_{\mathrm{t}}(\mathrm{A}) \subseteq$ $\overline{\mathcal{R}}_{\mathrm{j}}$ (A).

(iii) Using (i) and (ii) we have $\mathcal{B}_{\mathrm{t}}(\mathrm{A}) \subseteq \mathcal{B}_{\mathrm{j}}(\mathrm{A})$.

(iv) $\underline{\mathcal{R}}_{\mathrm{j}}(\mathrm{A}) \subseteq \underline{\mathcal{R}}_{\mathrm{t}}(\mathrm{A}) \Rightarrow\left|\underline{\mathcal{R}}_{\mathrm{j}}(\mathrm{A})\right| \leq\left|\underline{\mathcal{R}}_{\mathrm{t}}(\mathrm{A})\right|$ and

$\overline{\mathcal{R}}_{\mathrm{t}}(\mathrm{A}) \subseteq \overline{\mathcal{R}}_{\mathrm{j}}(\mathrm{A}) \Rightarrow\left|\overline{\mathcal{R}}_{\mathrm{t}}(\mathrm{A})\right| \leq\left|\overline{\mathcal{R}}_{\mathrm{j}}(\mathrm{A})\right|$, then we have

$\left|\frac{\mathcal{\mathcal { R }}_{\mathrm{j}}(\mathrm{A})}{\overline{\overline{\mathcal{R}}}_{\mathrm{j}}(\mathrm{A})}\right| \leq\left|\frac{\mathcal{\mathcal { R }}_{\mathrm{t}}(\mathrm{A})}{\overline{\overline{\mathcal{R}}}_{\mathrm{t}}(\mathrm{A})}\right| \Rightarrow \alpha_{\mathrm{j}}(\mathrm{A}) \leq \alpha_{\mathrm{t}}(\mathrm{A})$.

Proposition 2 . Let $\left(\mathrm{U}, \mathrm{R}, \xi_{\mathrm{j}}\right)$ be a $j$-neighborhood space and $\mathrm{A} \subseteq \mathrm{U}$. Then

(i) $\underline{\mathcal{R}}_{\mathrm{k}}(\mathrm{A}) \subseteq \underline{\mathcal{R}}_{\mathrm{t}}(\mathrm{A})$.

(ii) $\overline{\mathcal{R}}_{\mathrm{t}}(\mathrm{A}) \subseteq \overline{\mathcal{R}}_{\mathrm{k}}(\mathrm{A})$.

(iii) $\mathcal{B}_{\mathrm{t}}(\mathrm{A}) \subseteq \mathcal{B}_{\mathrm{k}}(\mathrm{A})$.

(iv) $\alpha_{k}(\mathrm{~A}) \leq \alpha_{\mathrm{t}}(\mathrm{A})$.

Proof. The proof is similar to the proof of Proposition 1 .

Proposition 3. Let $\left(\mathrm{U}, \mathcal{R}, \xi_{\mathrm{j}}\right)$ be a $j$-neighborhood space and $A \subseteq U$. Then

(i) $\underline{\mathcal{R}}_{\mathrm{s}}(\mathrm{A}) \subseteq \underline{\mathcal{R}}_{\mathrm{t}}(\mathrm{A})$.

(ii) $\overline{\mathcal{R}}_{\mathrm{t}}(\mathrm{A}) \subseteq \overline{\mathcal{R}}_{\mathrm{s}}(\mathrm{A})$.

(iii) $\mathcal{B}_{\mathrm{t}}(\mathrm{A}) \subseteq \mathcal{B}_{\mathrm{s}}(\mathrm{A})$. (iv) $\alpha_{s}\left(\right.$ A) $\leq \alpha_{t}($ A)

Proof. The proof is similar to the proof of Proposition 1

Remark 1 Let $\left(U, R, \xi_{j}\right)$ be a $j$-neighborhood space and $A, B \subseteq U$, then the following are not necessarily true.

(1) $\underline{\mathcal{R}}_{\mathrm{j}}(\mathrm{A})=\underline{\mathcal{R}}_{\mathrm{t}}(\mathrm{A})$,

(2) $\overline{\mathcal{R}}_{\mathrm{t}}(\mathrm{A})=\overline{\mathcal{R}}_{\mathrm{j}}(\mathrm{A})$,

(3) $\mathcal{B}_{\mathrm{t}}(\mathrm{A})=\mathcal{B}_{\mathrm{j}}(\mathrm{A})$,

(4) $\alpha_{\mathrm{j}}(\mathrm{A})=\alpha_{\mathrm{t}}(\mathrm{A})$,

(5) $\underline{\mathcal{R}}_{\mathrm{k}}(\mathrm{A})=\underline{\mathcal{R}}_{\mathrm{t}}(\mathrm{A})$,

(6) $\overline{\mathcal{R}}_{\mathrm{t}}(\mathrm{A})=\overline{\mathcal{R}}_{\mathrm{k}}(\mathrm{A})$,

(7) $\mathcal{B}_{\mathrm{t}}(\mathrm{A})=\mathcal{B}_{\mathrm{k}}(\mathrm{A})$,

(8) $\alpha_{\mathrm{k}}(\mathrm{A})=\alpha_{\mathrm{t}}(\mathrm{A})$,

(9) $\underline{\mathcal{R}}_{\mathrm{s}}(\mathrm{A})=\underline{\mathcal{R}}_{\mathrm{t}}(\mathrm{A})$,

(10) $\overline{\mathcal{R}}_{\mathrm{t}}(\mathrm{A})=\overline{\mathcal{R}}_{\mathrm{s}}(\mathrm{A})$,

(11) $\mathcal{B}_{\mathrm{t}}(\mathrm{A})=\mathcal{B}_{\mathrm{s}}(\mathrm{A})$,

(12) $\alpha_{s}(A)=\alpha_{t}(A)$.

The following example is employed as a counter example to show this remark.

Example 4 According to Examples 2 and 3, If $A=$ $\{a, b, d\}$, the twelve equalities in the above remark are not satisfied.

Considering a $j$-neighborhood space

$\left(\mathrm{U}, \mathrm{R}, \xi_{\mathrm{j}}\right)$, Propositions 1,2 , and 3 prove that the

approximations of sets using the operators $\underline{\mathcal{R}}_{t}$ and $\overline{\mathcal{R}}_{t}$ are more accurate than the approximations obtained by using the operators $\underline{\mathcal{R}}_{j}, \overline{\mathcal{R}}_{j}$ or $\underline{\mathcal{R}}_{k}, \overline{\mathcal{R}}_{k}$ or $\underline{\mathcal{R}}_{s}, \overline{\mathcal{R}}_{s}$. For this reason, we study the properties of $\underline{\mathcal{R}}_{t}$ and $\overline{\mathcal{R}}_{t}$ in the next proposition.

Proposition 4 Let $\left(\mathrm{U}, \mathrm{R}, \xi_{\mathrm{j}}\right)$ be a $j$-neighborhood space and $\mathrm{A}, \mathrm{B} \subseteq \mathrm{U}$. Then

$\left(\mathcal{L}_{1}\right) \underline{\mathcal{R}}_{t}(A) \subseteq A$.

$\left(\mathcal{L}_{2}\right) \underline{\mathcal{R}}_{t}(U) \subseteq U$.

$\left(\mathcal{L}_{3}\right) \underline{\mathcal{R}}_{t}(\emptyset)=\emptyset$.

$\left(\mathcal{L}_{4}\right) A \subseteq B \Rightarrow \underline{\mathcal{R}}_{t}(A) \subseteq \underline{\mathcal{R}}_{t}(B)$.

$\left(\mathcal{L}_{5}\right) \underline{\mathcal{R}}_{t}(A \cap B) \subseteq \underline{\mathcal{R}}_{t}(A) \cap \underline{\mathcal{R}}_{t}(B)$.

$\left(\mathcal{L}_{6}\right) \underline{\mathcal{R}}_{t}(A \cup B) \supseteq \underline{\mathcal{R}}_{t}(A) \cup \underline{\mathcal{R}}_{t}(B)$.

$\left(\mathcal{L}_{7}\right) \underline{\mathcal{R}}_{t}(A)=\left(\overline{\mathcal{R}}_{t}\left(A^{\mathrm{C}}\right)\right)^{\mathrm{C}}$.

$\left(\mathcal{U}_{1}\right) A \subseteq \overline{\mathcal{R}}_{t}(A)$.

$\left(\mathcal{U}_{2}\right) \overline{\mathcal{R}}_{t}(U)=U$

$\left(\mathcal{U}_{3}\right) \overline{\mathcal{R}}_{t}(\emptyset)=\emptyset$.

$\left(\mathcal{U}_{4}\right) A \subseteq B \Rightarrow \overline{\mathcal{R}}_{t}(A) \subseteq \overline{\mathcal{R}}_{t}(B)$.

$\left(\mathcal{U}_{5}\right) \overline{\mathcal{R}}_{t}(A \cap B) \subseteq \overline{\mathcal{R}}_{t}(A) \cap \overline{\mathcal{R}}_{t}(B)$. 
$\left(U_{6}\right) \overline{\mathcal{R}}_{t}(A \cup B) \supseteq \overline{\mathcal{R}}_{t}(A) \cup \overline{\mathcal{R}}_{t}(B)$.

$\left(U_{7}\right) \overline{\mathcal{R}}_{t}(A)=\left(\underline{\mathcal{R}}_{t}\left(A^{\mathrm{C}}\right)\right)^{\mathrm{C}}$.

$(\mathcal{L U}) \underline{\mathcal{R}}_{t}(A) \subseteq \overline{\mathcal{R}}_{t}(A)$.

Proof. The proof of $\left(\mathcal{L}_{1}\right),\left(\mathcal{L}_{2}\right),\left(\mathcal{L}_{3}\right),\left(\mathcal{U}_{1}\right),\left(\mathcal{U}_{2}\right)$ and $\left(\mathcal{U}_{3}\right)$ follows directly from Definition 7.

$\left(\mathcal{L}_{4}\right) \quad$ Let $\quad A \subseteq B$ and $p \in \underline{\mathcal{R}}_{\mathrm{t}}(A)$, then $\quad p \in$ A such that $\exists N_{t}(p) \neq \varnothing, N_{t}(p) \subseteq A$. Thus $p \in A \subseteq$ $B$ such that $\exists N_{t}(p) \neq \varnothing, N_{t}(p) \subseteq A \subseteq B$. Hence $p \in \underline{\mathcal{R}}_{\mathrm{t}}(B)$ and so $\underline{\mathcal{R}}_{\mathrm{t}}(A) \subseteq \underline{\mathcal{R}}_{\mathrm{t}}(B)$. Therefore, $A \subseteq$ $B \Rightarrow \underline{\mathcal{R}}_{\mathrm{t}}(A) \subseteq \underline{\mathcal{R}}_{\mathrm{t}}(B)$.

$\left(\mathcal{U}_{4}\right)$ Let $A \subseteq B$ and $p \in \overline{\mathcal{R}}_{t}(A)$, then we have:

(1) $p \in A \Rightarrow p \in A \subseteq B \Rightarrow p \in B \subseteq \overline{\mathcal{R}}_{t}(B) \Rightarrow p$

$$
\in \overline{\mathcal{R}}_{t}(B)
$$

(2) $p \in A^{\mathrm{C}}$.Then $\quad p \in \overline{\mathcal{R}}_{t}(A) \Rightarrow \forall N_{t}(p), N_{t}(p) \cap$ $A \neq \emptyset$. Since $A \subseteq B$, we have $\forall N_{t}(p), N_{t}(p) \cap B \neq$ $\emptyset$ and hence we have two cases:

(i) $p \in B-A \Rightarrow p \in B \Rightarrow p \in \overline{\mathcal{R}}_{t}(B)$.

(ii) $p \in B^{C}$. So $\forall N_{t}(p), N_{t}(p) \cap B \neq \varnothing \Rightarrow p \in$ $\overline{\mathcal{R}}_{t}(B)$.

Hence, by (1) and (2), we have $A \subseteq B \Rightarrow \overline{\mathcal{R}}_{t}(A) \subseteq$ $\overline{\mathcal{R}}_{t}(B)$.

$\left(\mathcal{L}_{5}\right)$ Let $p \in \underline{\mathcal{R}}_{t}(A \cap B) \Rightarrow p \in(A \cap B), \exists N_{t}(p) \neq$ $\emptyset, N_{t}(p) \subseteq(A \cap B)$

$\Longrightarrow p \in A, \exists N_{t}(p) \neq \varnothing, N_{t}(p) \subseteq A \wedge p$

$$
\in B, \exists N_{t}(p) \neq \varnothing, N_{t}(p) \subseteq B
$$

$\Rightarrow p \in \underline{\mathcal{R}}_{t}(A) \quad \wedge p \in \underline{\mathcal{R}}_{t}(B) \Rightarrow p \in \underline{\mathcal{R}}_{t}(A) \cap$

$\underline{\mathcal{R}}_{t}(B)$.

$\left(\mathcal{U}_{5}\right)(A \cap B) \subseteq A \Rightarrow \overline{\mathcal{R}}_{t}(A \cap B) \subseteq \overline{\mathcal{R}}_{t}(A)$ and

$(A \cap B) \subseteq B$

$\Rightarrow \overline{\mathcal{R}}_{t}(A \cap B) \subseteq \overline{\mathcal{R}}_{t}(B)$. So $\quad \overline{\mathcal{R}}_{t}(A \cap B) \subseteq \overline{\mathcal{R}}_{t}(A) \cap$ $\overline{\mathcal{R}}_{t}(B)$.

$\left(\mathcal{L}_{6}\right) A \subseteq(A \cup B) \Rightarrow \underline{\mathcal{R}}_{t}(A) \subseteq \underline{\mathcal{R}}_{t}(A \cup B)$ and $B \subseteq$ $(A \cup B)$

$\Rightarrow \underline{\mathcal{R}}_{t}(B) \subseteq \underline{\mathcal{R}}_{t}(A \cup B) . \quad$ Hence $\quad \underline{\mathcal{R}}_{t}(A \cup B) \supseteq$ $\underline{\mathcal{R}}_{t}(A) \cup \underline{\mathcal{R}}_{t}(B)$.

$\left(\mathcal{U}_{6}\right)$ Let $p \notin \overline{\mathcal{R}}_{t}(A \cup B)$, then $p \notin(A \cup B)$ and $p \in(A \cup B)^{C}, \exists N_{t}(p), N_{t}(p) \cap(A \cup B)=\emptyset$. So $p \in\left(A^{\mathrm{C}} \cap B^{\mathrm{C}}\right), \exists N_{t}(p),\left(N_{t}(p) \cap A\right) \cup\left(N_{t}(p) \cap\right.$

$A)=\emptyset$. Thus

$p \in A^{\mathrm{C}}, \exists N_{t}(p), N_{t}(p) \cap A=\varnothing \wedge p \in$

$B^{\complement}, \exists N_{t}(p), N_{t}(p) \cap B=\emptyset$

$\Rightarrow p \notin \overline{\mathcal{R}}_{t}(A) \wedge p \notin \overline{\mathcal{R}}_{t}(B) \Rightarrow p \notin\left(\overline{\mathcal{R}}_{t}(A) \cup\right.$

$\left.\overline{\mathcal{R}}_{t}(B)\right)$.

$\left(\mathcal{L}_{7}\right) \quad$ Let $\quad p \in \underline{\mathcal{R}}_{t}(A) \Leftrightarrow p \in A, \exists N_{t}(p) \neq$

$\emptyset, N_{t}(p) \subseteq A \Leftrightarrow p \in\left(A^{\mathrm{C}}\right)^{\mathrm{C}}, \exists N_{t}(p) \neq \emptyset, N_{t}(p) \cap$

$A^{\mathrm{C}}=\varnothing$

$\Leftrightarrow P \notin \overline{\mathcal{R}}_{t}\left(A^{\mathrm{C}}\right) \Leftrightarrow P \in\left(\overline{\mathcal{R}}_{t}\left(A^{\mathrm{C}}\right)\right)^{\mathrm{C}}$.

Hence $\underline{\mathcal{R}}_{t}(A)=\left(\overline{\mathcal{R}}_{t}\left(A^{\mathrm{C}}\right)\right)^{\mathrm{C}}$.

$\left(\mathcal{U}_{7}\right)$ By substituting $A^{\mathrm{C}}$ for $A$ in $\left(\mathcal{L}_{7}\right)$ we have $\overline{\mathcal{R}}_{t}(A)=\left(\underline{\mathcal{R}}_{t}\left(A^{\mathrm{C}}\right)\right)^{\mathrm{C}}$.

(LU) Obviously, by $\left(\mathcal{L}_{1}\right)$ and $\left(\mathcal{U}_{1}\right)$ we get $\underline{\mathcal{R}}_{t}(A) \subseteq$ $\overline{\mathcal{R}}_{t}(A)$.

\section{Conclusions}

In this paper, we introduce three types of neighborhood systems in a $j$-neighborhood space, namely, the k- neighborhood system, s- neighborhood system and t- neighborhood system. Using these three neighborhood systems, we define the lower and upper rough set approximations. Comparisons between the accuracy of these three types of approximations are superimposed.

Propositions 1, 2, and 3 prove that the approximations of sets using the operators $\underline{\mathcal{R}}_{t}$ and $\overline{\mathcal{R}}_{t}$ are more accurate than the approximations obtained by using the operators $\underline{\mathcal{R}}_{j}, \overline{\mathcal{R}}_{j}$ or $\underline{\mathcal{R}}_{k}, \overline{\mathcal{R}}_{k}$ or $\underline{\mathcal{R}}_{s}, \overline{\mathcal{R}}_{s}$. That is, the best approximations are those based on the t- neighborhood systems.

Considering the $j$-neighborhood space, this study provides a method to improve the accuracy of rough set approximations by using the t- neighborhood system.

\section{References}

[1] Abd El-Monsef, M.E.; Embaby, O. A ; El-Bably, M.K.: Comparison between rough set approximations based on different topologies, Int. J. Granular Computing, Rough Sets and Intelligent Systems, Vol. 3, No. 4, (2014) 292-305.

[2] Abd El-Monsef, M.E.; Salama, A.S.; Embaby,O.A.: Granular computing using mixed neighborhood systems, Jour. of Inst. of Mathematics \& Computer Science (Computer Science Ser.) Vol.20, No.2 (2009) 233-243. 
[3] Allam, A. A., Bakeir, M. Y. and Abo-Tabl, E. A.: New approach for basic rough set concepts, in: Rough Sets, Fuzzy Sets, Data Mining, and Granular Computing, Lecture Notes in Artificial Intelligence 3641, D. Slezak, G. Wang, M. Szczuka, I. Dntsch,Y. Yao (Eds.), Springer Verlag GmbH ,Regina, (2005), 64-73.

[4] De Baets, B.; Kerre, E.: A revision of bandlerkohout compositions of relations, Mathematic Pannonica, 4/1, (1993), 59-78.

[5] Galton, A.: A generalized topological view of motion in discrete space, Theoretical Computer Sciences, volume 305, issues 1-3,(2003), 111-134.

[6] Kortelainen, J.: On the relationship between modified sets, topological spaces and rough sets, Fuzzy Sets and Systems, 61 (1994), 91-95.

[7] Largeron, C.; Bonnevay, S.: A pretopological approach for structural analysis, Information Sciences, 144 (2002), 185-196.

[8] Lashin, E.F.; Kozae, A.M.; Abo Khadra, A.A.; Medhat, T.: Rough set theory for topological spaces, International Journal of Approximate Reasoning, 40 (12) (2005), 35-43.

[9] Lin, T.Y.: Granular computing on binary relations I: Data mining and neighborhood systems, II: Rough set representations and belief functions, in: Rough Sets in Knowledge Discovery 1, Polkowski, L., and Skowron, A. (Eds.), Physica-Verlag,Heidelberg, (1998), 107 -140.

[10] Liu, G.L.; Zhu, W.: The algebraic structures of generalizedroughset theory, Information Sciences, 178 (2008), 4105-4113.

[11] Pawlak, Z.: Rough sets, Int. J. of Information and Computer Sciences, 11 (5) (1982), 341-356.

[12] Pawlak, Z.: Rough sets theoretical aspects of reasoning about data, vol. 9, KluwerAcademic Publishers, Dordrecht, 1991.

[13]Pawlak, Z.;Skowron, A.: Rough sets: some extensions, Information Sciences, 177(2007), 2840 .

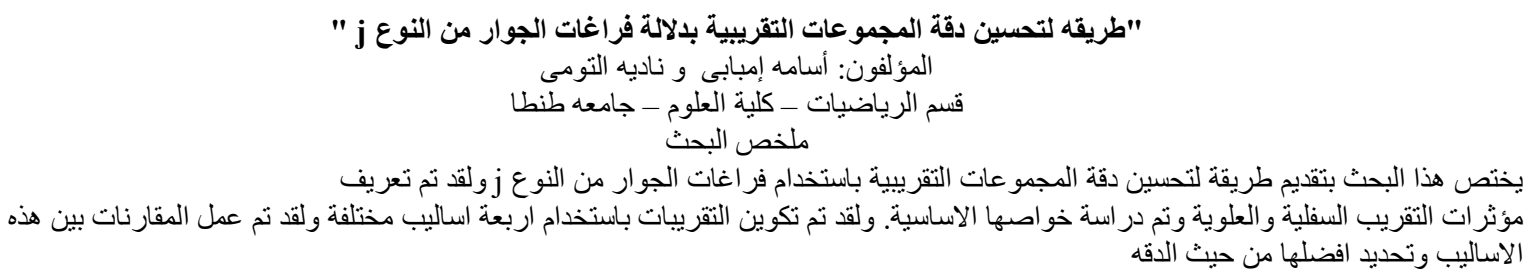

[14]Skowron, A.; Stepaniuk, J.: Tolerance approximation spaces, Fundamenta Informaticae, 27 (1996), 245-253.

[15] Slowinski, R.; Vanderpooten, D.: A generalized definition of rough approximations based on similarity, IEEE Transactions on and Data Engineering, 12 (2) (2000), 331-336.

[16] Stadler, B.M.R.; Stadler, P.F.: Generalized topological spaces in evolutionary theory and combinatorial chemistry, J. Chem. Inf. Comp. Sci. 42 (2002), 577-585.

[17] Stadler, B.M.R.; Stadler, P.F.: The topology of evolutionary Biology, Ciobanu, G., and Rozenberg, G. (Eds.): Modeling in Molecular Biology, Springer Verlag, Natural Computing Series, (2004), 267-286.

[18] Wu, W.Z.; Zhang, W.X.: Rough set approximations vs. measurable spaces,in: IEEE International Conference on Granular Computing, (2006), 329-332.

[19] Yao, Y.Y.: Relational interpretations of neighborhood operators and rough set approximation operators, Information Sciences, 111 (1-4) (1998), 239-259.

[20] Yao, Y.Y.: Constructive and algebraic methods of theory of rough sets, Information Sciences, 109 (1998), 21-47.

[21] Yao, Y.Y.:Rough sets, neighborhood systems and granular computing, Proceedings of the IEEE Canadian Conference on Electrical and Computer Engineering,(1999), 1553-1559.

[22] Zhang, H.; Ouyang, Y.; Wang, Z.:Note on "Generalized roughsets based on reflexive and transitive relations", Information Sciences, 179 (2009), 471-473. 